\title{
Endurance of erythrocyte series in chemotherapy
}

\author{
SORIN SĂFTESCU ${ }^{1}$, DOREL POPOVICI ${ }^{1}$, CRISTINA OPREAN $^{1}$, ALINA NEGRU ${ }^{2}$, ADINA CROITORU $^{3}$, \\ MIHAIL ZEMBA $^{4}$, IONELA YASAR ${ }^{4}$, MARIA PREDA $^{5}$ and ȘERBAN NEGRU ${ }^{1}$ \\ Departments of ${ }^{1}$ Oncology and ${ }^{2}$ Cardiology, 'Victor Babeș' University of Medicine and Pharmacy, \\ 300041 Timisoara; ${ }^{3}$ Department of Medical Oncology, Fundeni Clinical Institute, 022328 Bucharest; \\ ${ }^{4}$ Department of Ophthalmology, 'Carol Davila' University of Medicine and Pharmacy, 050474 Bucharest; \\ ${ }^{5}$ Department of Ophthalmology, 'Victor Babeș' University of Medicine and Pharmacy, 300041 Timisoara, Romania
}

Received August 25, 2020; Accepted September 24, 2020

DOI: $10.3892 / \mathrm{etm} .2020 .9344$

\begin{abstract}
Hematopoietic bone marrow toxicity is most often the limiting factor for chemotherapy doses. Increasing the intensity of chemotherapy doses (higher doses or more frequent administration) would improve antitumor effects, but the hematological toxicity does not allow these dose increases. This study evaluated the impact of chemotherapies on the parameters belonging to the red blood cell series in the hemogram and aimed to identify some particular evolution profiles. We selected 855 evaluations performed before the administration of chemotherapy belonging to the treatments initiated during the period December 2018-February 2020, containing 5-fluorouracil, cisplatin, docetaxel, epirubicin or pemetrexed. The data of the 644 evaluations related to the cycles 1-4 of chemotherapy were subject to this processing. The average relative loss of hemoglobin is $-11 \%$ after the first three cycles of treatment, with statistically significant differences in hemoglobin levels in favor of men. There are risk factors associated with higher average losses, such as age $<50$ years or $>65$ years (statistically significant), body mass index (BMI) $>25$, cisplatin treatment (insufficient number of cases to reach statistical significance).
\end{abstract}

\section{Introduction}

Physiology of erythropoiesis. The bone marrow located in the membranous bones (vertebrae, ribs, skull, sternum, shoulder blades and pelvis) is responsible for erythropoiesis in adult individuals.

The stages of differentiation of the red line starts from multipotent stem cells (also called hemocytoblasts), goes through common multipotent myeloid progenitors, unipotent stem

Correspondence to: Dr Dorel Popovici, Department of Oncology, 'Victor Babeș' University of Medicine and Pharmacy, Piața Eftimie Murgu 2, 300041 Timisoara, Romania

E-mail: dorelpopovici@gmail.com

Key words: haemoglobin, erythrocytes, chemotherapy, bone marrow, cancer cells, proerythroblasts (also called pronormoblast), basophilic normoblasts (also called erythroblast-secretes erythroferrone that inhibits the hepcidin secretion), intermediate normoblastpolychromatophilic, orthochrome normoblast (the nucleus is expelled) and reticulocytes (normally accounts for $1 \%$ of blood cells and lasts for 1-2 days until maturation to erythrocytes). In addition to the nucleus, reticulocytes do not possess mitochondria, nor Golgi apparatus or endoplasmic reticulum (1).

The duration of erythropoiesis was estimated at seven days. The mature cells of the erythroid series are anucleated in mammals, but nucleated in the vast majority of the other vertebrates. The discoid-biconvex form is not universally widespread in mammals, with species having small spherical cells, oval cells, even fusiform, polygonal or angled cells (2).

The progenitor cells undergo a marked reduction in cell volume starting at $900 \mathrm{fl}$ for precursors and ending with $95 \mathrm{fl}$ for mature erythrocytes. The reticulocyte is still capable of hemoglobin synthesis, although it is anucleated. Erythropoiesis is conditioned by the supply of erythropoietin (of renal and hepatic origin), folate (vitamin B9) and cyanocobalamin (vitamin B12). The deficiency of any of these substances results in reticulocytopenia. Macrophages from the hematogenous marrow under the action of hepcidin release the sequestered iron to be provided to the erythrocyte progenitors for hem synthesis $(3,4)$.

The average lifespan of an erythrocyte is 120 days and, every day, 200 billion red blood cells are released into circulation and 'withdrawn' respectively (1). Subject to capillary deformation, red blood cells can release ATP and nitric oxide with a vasodilator role, and upon release of hemoglobin-bound oxygen, they release S-nitroso-thiols, which also have a vasodilator role (5-7).

Erythrocyte parameters: mean corpuscular volume (MCV): Normal values between 80 and $100 \mathrm{fl}$ (according to other sources 80-94 fl); lower values appear in iron deficiency and thalassemia. Higher values occur in the folate or B12 deficiency, as well as after chemotherapy. Normal values are present in aplastic anaemia, chronic disease anaemia or post-chemotherapy anaemia.

$\mathrm{MCH}$ mean corpuscular hemoglobin, the average hemoglobin load, represents the average amount of hemoglobin per erythrocyte. Normal values are between 27 and $31 \mathrm{pg}$. Lower values occur in hypochromic anaemia and higher 
values in primary or post-chemotherapy vitamin B9 or B12 deficiency (8-10).

RDW-CV red blood cell distribution width is a measure of red cell volume variability (standard deviation of MCV relative to $\mathrm{MCV}$ ). Normal values are between 11.5 and $14.5 \%$. Increased values occur in megalocytic anemias (2/3 of cases), and low values are constant in iron deficiency anaemia. The high values of RDW-CV are synonymous with anisocytosis (11).

MCHC medium corpuscular hemoglobin concentration, the average hemoglobin concentration in erythrocytes, correlates the hemoglobin cell content with the volume of erythrocytes. It is expressed in $\mathrm{g} / \mathrm{dl}$ (as in blood hemoglobin) and has normal values of $34 \pm 2 \mathrm{~g} / \mathrm{dl}(9)$.

Hematological toxicity of chemotherapy. Hematopoietic bone marrow toxicity is most often the limiting factor for chemotherapy doses. Increasing the intensity of chemotherapy doses (higher doses or more frequent administration) would allow improved antitumor effects, but the hematological side effects do not allow such dose increases. The possibility of transfusion for the erythroid concentrates results in a limited effect of anaemia in the intensity of the prescribed chemotherapy regimen. However, the need for multiple transfusions and the associated symptomatology of mild and the medium anaemia decrease the quality of life and remain valid problems in oncology.

Conversely, studies have shown that chemotherapy regimens associated with more profound neutropenia and thrombocytopenia are associated with better survival rates in colorectal cancers, which is not valid for anaemia, that has a worse prognosis. Myelosuppression during chemotherapy could be a biological measure of drug efficacy. It could also be a predictive factor for treatment success under conditions of interindividual variability in the activation/metabolization of chemotherapeutic agents (12). Other studies have shown a favorable predictive effect on survival and response to treatment associated with increased MCV (macrocytosis) during capecitabine treatment in patients with advanced gastric cancer (13).

The possibility of using granulocyte growth factors (G-CSF-Filgrastim) for neutropenia prophylaxis during chemotherapy allows the preservation of chemotherapy dose intensity. However, studies have shown that deviation of the myeloid line toward granulocyte precursors exposes patients to more severe anaemia when their neoplasm was treated with epirubicin and cyclophosphamide (14).

The classification of anaemia severity as an adverse effect of the treatments (including chemotherapy) defines grade 1 anaemia at values between $10 \mathrm{~g} / \mathrm{dl}$ and the lower limit of the normal according to sex. Grade 2 is between 9.9 and $8.0 \mathrm{~g} / \mathrm{dl}$, grade 3 has values of hemoglobin between 7.9 and $6.5 \mathrm{~g} / \mathrm{dl}$ and grade $4<6.5 \mathrm{~g} / \mathrm{dl}$.

Nevertheless, the correlation of the anaemia symptomatology with hemoglobin values is quite weak. From all the symptoms, fatigue seems to be the cardinal complaint. This symptom is rarely evaluated and addressed therapeutically, but it is one of the main causes for the refusal of chemotherapy. Anaemia also correlates with the ability to perform various household/professional tasks, as well as with the ability and willingness to participate in recreational activities. In a study that evaluated patients with non-small cell lung cancer, grade 2 anaemia was present in $30 \%$ of the patients treated after the first cycle of chemotherapy and in $59 \%$ of the patients after 4 cycles of chemotherapy. Elderly patients with neoplasms may exhibit anaemia-related symptoms at higher hemoglobin levels compared with younger patients. The proportion of neoplastic patients requiring transfusions is maximal in the case of hematological disorder (lymphomas), lung cancer, gynecological and genito-urinary cancers; $50-60 \%$ of these patients need a blood transfusion (15-18).

Treatment options for anaemia caused by chemotherapy include iron, B9 and B12 - in case of documented deficiencies in these substances, further stimulation of erythroid series using erythropoietin, transfusion of cellular concentrate (erythrocyte concentrate/erythrocyte mass) and rarely whole blood; in case of emergencies (acute anemias due to bleeding from chronic anaemia), in addition to erythrocyte mass transfusion, the contribution of crystalloid solutions and plasma expanders can be considered.

Objectives. The multitude of patients treated with chemotherapy, the increasing number of treatment lines that have become available (many of which include chemotherapy) and the legitimate desire to control the hematological adverse effects of chemotherapy reveal the necessity for more detailed knowledge of the impact of cytostatic treatments on hematopoiesis. This study evaluated the impact of chemotherapies on the current parameters analyzed in the hemogram, as well as to identify particular evolution profiles during the treatment.

\section{Materials and methods}

The retrospective study was based on 855 clinical and biological assessments before chemotherapy administration, selected from December 2018 to February 2020 (chemotherapy regimens including 5-fluorouracil, cisplatin, docetaxel, epirubicin and pemetrexed). Only the patients in which the chemotherapy was initiated during the study period were included. Patients with chemotherapy in progress in December 2018 were excluded. In total, 523 administrations were performed in men and 332 in women. The number of individual patients is 250 persons, of which 150 men and 100 women. The average age of the group is 59.46 years (58.49 for men, 61.03 for women). Height, weight, body mass index (BMI), complete blood count details, and creatinine were retrieved at each assessment performed before the initiation of chemotherapy and then before cycles 2, 3 and 4 of chemotherapy (in fact the data are those pre-chemotherapy and after the cycle 1 , after cycle two and after administration of cycle three before administration of cycle 4). No data were processed for subsequent cycles because the number of such cases is too small to allow conclusions. The fluoropyrimidine and cisplatin-based chemotherapies were accurately processed because the available cases were numerous.

\section{Results}

The number of administrations performed for each cycle indicates a progressive decrease from one series of treatments to another (fewer patients reached the next cycle due to intolerance, disease progression or refusal of treatment). The 
Table I. Distribution of administrations by treatment cycles.

\begin{tabular}{lcccccccccc}
\hline Cycle number & 1 & 2 & 3 & 4 & 5 & 6 & 7 & 8 & 9 & 10 \\
\hline Chemo administrations & 254 & 172 & 128 & 90 & 49 & 40 & 24 & 20 & 16 & 11 \\
\hline
\end{tabular}

Table II. Evolution of the mean value of hemoglobinemia (g/dl) under chemotherapy.

\begin{tabular}{lccccc}
\hline Cycle number & 1 & 2 & 3 & 4 & $\Delta$ C4 vs. C1\% \\
\hline Medium Hb & 12.42 & 11.67 & 11.32 & 11.03 & -11 \\
Medium Hb, males & 12.77 & 11.95 & 11.51 & 11.22 & -12 \\
Medium Hb, females & 11.9 & 11.24 & 10.97 & 10.7 & -10 \\
\hline
\end{tabular}

Table III. Evolution of the average hemoglobin value $(\mathrm{g} / \mathrm{dl})$ by age groups.

\begin{tabular}{lccccc}
\hline Cycle number & 1 & 2 & 3 & 4 & $\Delta$ C4 vs. C1\% \\
\hline Medium Hb, age $<50$ years & 12.95 & 12.38 & 11.87 & 11.14 & -14 \\
Medium Hb, age $=50$-65 years & 12.41 & 11.71 & 11.45 & 11.42 & -8 \\
Medium Hb, age MI $\geq 65$ years & 12.15 & 11.24 & 10.86 & 10.45 & -14
\end{tabular}

maximum number of cycles of chemotherapy per person during the period studied was 27 (Table I).

Out of the 855 administrations, 231 were for lung, 146 for esophageal and gastric, 145 for colorectal, 52 for ENT, 47 for pancreatic, 36 for breast, 30 for bladder and 28 for testicular neoplasms.

The most interesting parameter is the evolution of average hemoglobin values for each administration cycle that reveals different starting levels. We observed higher average starting values for men (with average onset in the area of mild anaemia), but with a slightly higher relative decrease in males. Relative hemoglobin loss is $-11 \%$ after three cycles of chemotherapy. The most abrupt decrease was recorded after the first administration of chemotherapy. The decrease in $\mathrm{Hb}$ from $\mathrm{C} 1$ to $\mathrm{C} 4$ was statistically significant (t-test, $\mathrm{P}<0.0001)$. Because of the progressive decrease of patients after each treatment cycle, statistical significance $(\mathrm{P}=0.013)$ for the difference of $\mathrm{Hb}$ in genders was demonstrated up to C2 (Table II and Fig. 1).

Starting values are higher for patients $<50$ years of age and decrease progressively with age. The most significant decreases during treatment are in the area of patients of extreme age $(<50$ or $>65)$. The differences between the group $<50$ and 50-65 years are statistically significant at $\mathrm{C} 4$ $(\mathrm{P}=0.0025)$ and between the group $<50$ and $>65$ years only at $\mathrm{C} 3(\mathrm{P}=0.0161)$ for the same reasons for a lower number of cases at C4 (Table III and Fig. 2).

A regular or low BMI is associated with lower starting hemoglobin, overweight patients are initiated with the best hemoglobin capital, but suffer the most considerable relative decrease. The differences are statistically significant at $\mathrm{C} 3$ between persons with BMI $<25 \mathrm{~kg} / \mathrm{m}^{2}$ and those with BMI between 25 and $29 \mathrm{~kg} / \mathrm{m}^{2}(\mathrm{P}=0.0277)$ (Table IV).

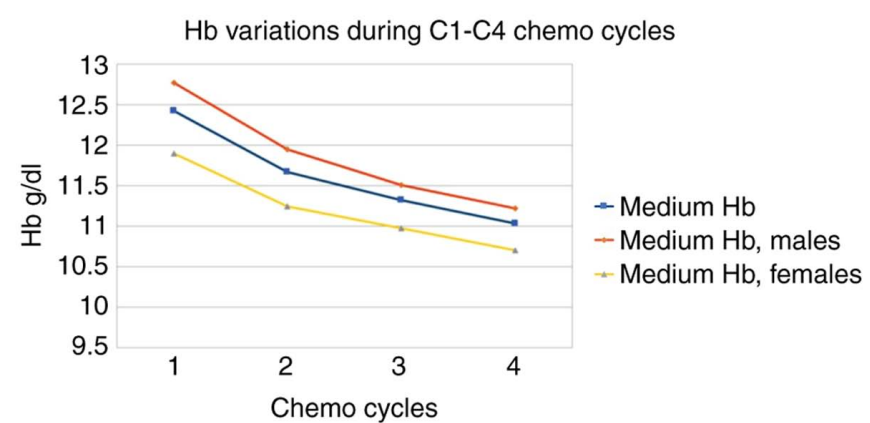

Figure 1. The evolution of the mean value of hemoglobinemia under chemotherapy.

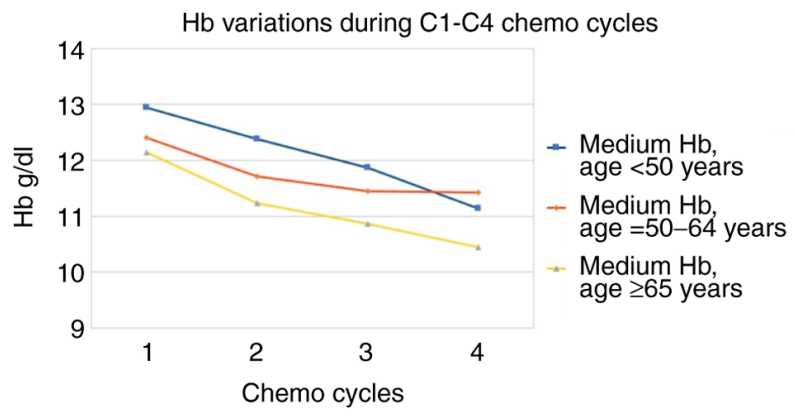

Figure 2. The evolution of the average hemoglobin value by age groups.

Fluoropyrimidine (5-fluorouracil)-based chemotherapy induces less severe anaemia compared with that caused 
Table IV. Evolution of mean hemoglobin value $(\mathrm{g} / \mathrm{dl})$ by body mass index $\left(\mathrm{BMI}, \mathrm{kg} / \mathrm{m}^{2}\right)$ groups.

\begin{tabular}{lccccc}
\hline Cycle number & 1 & 2 & 3 & 4 & $\Delta$ C4 vs. C1 \% \\
\hline Medium Hb, BMI $<25 \mathrm{~kg} / \mathrm{m}^{2}$ & 11.98 & 11.23 & 11.03 & 10.88 & -9 \\
Medium $\mathrm{Hb}, \mathrm{BMI}=25-29 \mathrm{~kg} / \mathrm{m}^{2}$ & 12.98 & 12.14 & 11.81 & 11.31 & -13 \\
Medium Hb, BMI $\geq 30 \mathrm{~kg} / \mathrm{m}^{2}$ & 12.53 & 12.01 & 11.42 & 11.14 & -11
\end{tabular}

Table V. Evolution of the average hemoglobin value $(\mathrm{g} / \mathrm{dl})$ according to different chemotherapies.

\begin{tabular}{lccccc}
\hline Cycle number & 1 & 2 & 3 & 4 & $\Delta$ C4 vs. C1 \% \\
\hline 5-fluorouracil & 11.66 & 11.25 & 11.14 & 10.99 & -6 \\
Cisplatin & 12.68 & 11.84 & 11.32 & 10.95 & -14
\end{tabular}

Table VI. Evolution of mean hemoglobin value $(\mathrm{g} / \mathrm{dl})$ according to patient height.

\begin{tabular}{lccccc}
\hline Cycle number & 1 & 2 & 3 & 4 & $\Delta$ C4 vs. C1 \% \\
\hline Medium $\mathrm{Hb}, \mathrm{h}<160 \mathrm{~cm}$ & 12.25 & 11.59 & 11.11 & 10.71 & -13 \\
Medium $\mathrm{Hb}, \mathrm{h}=160-179 \mathrm{~cm}$ & 12.37 & 11.59 & 11.27 & 11.05 & -11 \\
Medium $\mathrm{Hb}, \mathrm{h} \geq 180 \mathrm{~cm}$ & 13.18 & 12.34 & 11.98 & 11.53 & -13 \\
\hline
\end{tabular}

Table VII. Evolution of mean hemoglobin value (g/dl) according to creatinine level.

\begin{tabular}{lllllr}
\hline Cycle number & 1 & 2 & 3 & 4 & $\Delta \mathrm{C} 4 \mathrm{vs} . \mathrm{C} 1 \%$ \\
\hline Medium $\mathrm{Hb}$, creatinine $<0.6 \mathrm{mg} / \mathrm{dl}$ & 11.4 & 11.04 & 11.02 & 11.12 & -2 \\
Medium $\mathrm{Hb}$, creatinine $=0.6-1.09 \mathrm{mg} / \mathrm{dl}$ & 12.85 & 12.06 & 11.48 & 11.15 & -13 \\
Medium $\mathrm{Hb}$, creatinine $\geq 1.1 \mathrm{mg} / \mathrm{dl}$ & 11.64 & 10.76 & 10.39 & 10.44 & -10 \\
\hline
\end{tabular}

Table VIII. Evolution of the value of mean corpuscular volume (MCV, fL) by sex.

\begin{tabular}{lccccc}
\hline Cycle number & 1 & 2 & 3 & 4 & $\Delta C 4$ vs. C1 \% \\
\hline Male MCV & 86.36 & 86.18 & 86.7 & 88.13 & 2 \\
Female MCV & 85.89 & 85.84 & 86.96 & 88.63 & 3 \\
\hline
\end{tabular}

by cisplatin. The decrease in hemoglobin from $\mathrm{C} 1$ to $\mathrm{C} 4$ is statistically significant $(\mathrm{P}<0.0001)$ (Table $\mathrm{V}$ and Fig. 3).

Starting hemoglobin is proportional to the height of the patients, while the decreases of hemoglobinemia are slightly lower for the average height people (Table VI and Fig. 4).

Patients with normal low or under the lower limit serum creatinine start with the lowest hemoglobin capital, but suffer the least deterioration. The difference between the initial $\mathrm{Hb}$ values for the creatinine group $<0.6$ and the creatinine group between 0.6 and 1.1, respectively, are statistically significant $(\mathrm{P}<0.0001)$ (Table VII and Fig. 5).
Regarding the evolution of the MCV, the trend is upward, passing through a period of latency or a slight transient decrease (Table VIII). By age group, the highest increase of MCV occurs in young patients, without latency (Table IX and Fig. 6).

Regarding the different chemotherapeutics used, cisplatin causes a higher increase in MCV compared with 5-FU (Table $\mathrm{X}$ and Fig. 7). The variation of MCV, according to $\mathrm{BMI}$, revealed a minimum impact in the area of normal or overweight people and a maximum in the area of obese people (Table XI and Fig. 8). 


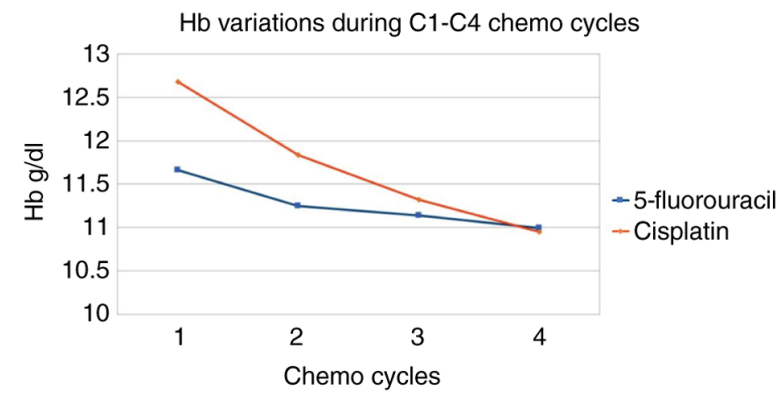

Figure 3. The evolution of the average hemoglobin value according to different chemotherapies.

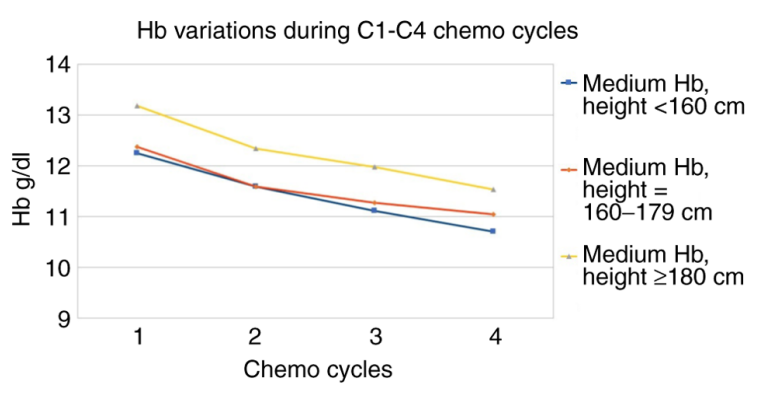

Figure 4. The evolution of the average hemoglobin value according to the height of the patients.

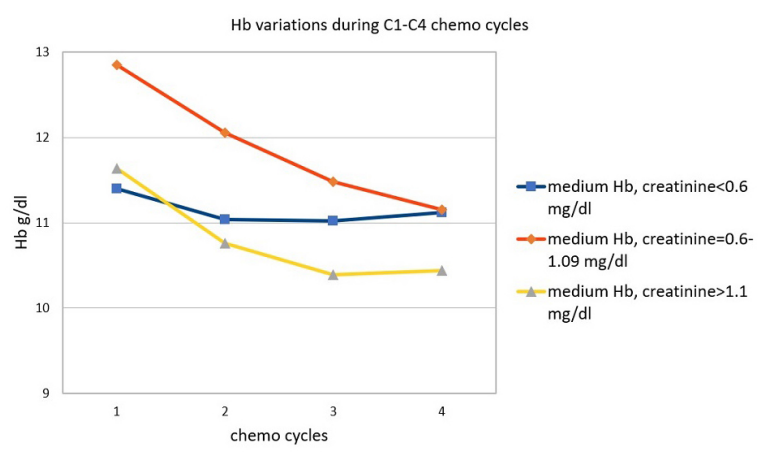

Figure 5. The evolution of the average hemoglobin value according to the level of creatinine.

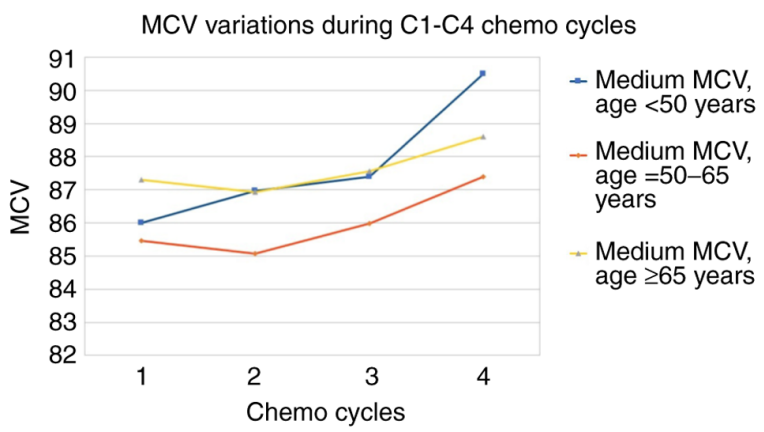

Figure 6. The evolution of the average value of the mean corpuscular volume $(\mathrm{MCV}, \mathrm{fL})$ by age group.

The evolution of the erythrocyte volume dispersion index (RDW-CV) is somewhat surprising regarding its reduction

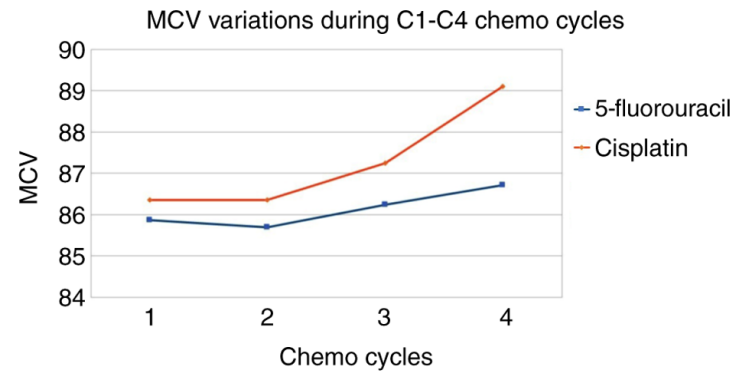

Figure 7. The evolution of the average value of mean corpuscular volume (MCV, fL) according to the type of cytostatic.

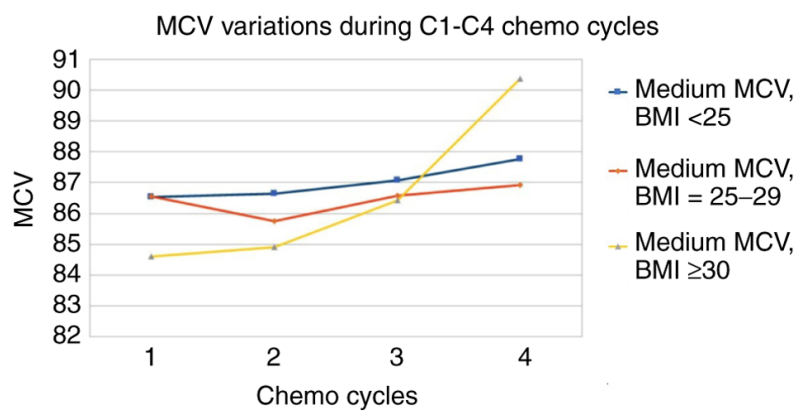

Figure 8 . The evolution of the average value of the mean corpuscular volume $(\mathrm{MCV}, \mathrm{fL})$ by body mass index $\left(\mathrm{BMI}, \mathrm{kg} / \mathrm{m}^{2}\right)$.

under chemotherapy, especially under cisplatin, despite the increase of average MCV (Table XII).

\section{Discussion}

The mean relative hemoglobin loss is $11 \%$ after three cycles of chemotherapy $(-1.39 \mathrm{~g} / \mathrm{dl})$, without the mean hemoglobin levels leaving the area of mild anaemia. The most abrupt decrease in hemoglobin is recorded after the first chemotherapy administration. No data were obtained for possible transfusions. Previous studies on cancer patients receiving chemotherapy revealed 43-54\% of patients having less than $9 \mathrm{~g} / \mathrm{dl}$ haemoglobin after 6 weeks of treatment. Also the percent of patients requiring transfusion reached up to $43 \%$ during chemotherapy (19).

By age groups: The most significant hemoglobin decreases during treatment are in the area of patients of extreme age $(<50$ or $>65)$. Chemotherapy dosages may be more 'courageous' in younger patients (fewer dose reductions). The highest increase in MCV occurs in young patients without latency. Studies on elderly lung cancer patients treated with chemotherapy demonstrated higher $\mathrm{Hb}$ values significantly associated with more favourable values of indexes measuring mental and functional capacity and depression (20).

Overweight patients start with the best hemoglobin value, but they also suffer the most considerable relative decrease. MCV growth appears most pronounced in obese people.

Fluoropyrimidine (5-fluorouracil)-based chemotherapy induces less severe anaemia compared with cisplatin. In addition, the impact of cisplatin on MCV is more significant.

People with low creatinine levels demonstrate the lowest level of initial hemoglobin but suffer the least deterioration. Other authors demonstrated that decreased creatinine 
Table IX. Evolution of the average value of mean corpuscular volume (MCV, fL) by age group.

\begin{tabular}{lccccc}
\hline Cycle number & 1 & 2 & 3 & 4 & $\Delta$ C4 vs. C1 \% \\
\hline Medium MCV, age $<50$ years & 86.01 & 86.97 & 87.39 & 90.49 & 5 \\
Medium MCV, age $=50$-65 years & 85.47 & 85.07 & 85.99 & 87.4 & 2 \\
Medium MCV, age $\geq 65$ years & 87.31 & 86.94 & 87.57 & 88.6 & 1 \\
\hline
\end{tabular}

Table X. Evolution of the mean value of mean corpuscular volume (MCV, fL) by cytostatic type.

\begin{tabular}{lccccc}
\hline Cycle number & 1 & 2 & 3 & 4 & $\Delta$ C4 vs. C1 \% \\
\hline 5-fluorouracil & 85.87 & 85.7 & 86.24 & 86.72 & 1 \\
Cisplatin & 86.36 & 86.36 & 87.25 & 89.1 & 3 \\
\hline
\end{tabular}

Table XI. Evolution of the average value of the mean corpuscular volume (MCV, $\mathrm{fL})$ by body mass index $\left(\mathrm{BMI}, \mathrm{kg} / \mathrm{m}^{2}\right)$.

\begin{tabular}{llllll}
\hline Cycle number & 1 & 2 & 3 & 4 & $\Delta$ C4 vs.C1\% \\
\hline Medium MCV, BMI $<25 \mathrm{~kg} / \mathrm{m}^{2}$ & 86.53 & 86.63 & 87.07 & 87.77 & 1 \\
Medium MCV, BMI $=25-29 \mathrm{~kg} / \mathrm{m}^{2}$ & 86.56 & 85.76 & 86.57 & 86.92 & 0 \\
Medium MCV, BMI $\geq 30 \mathrm{~kg} / \mathrm{m}^{2}$ & 84.6 & 84.92 & 86.43 & 90.37 & 7 \\
\hline
\end{tabular}

Table XII. Evolution of the mean value of RDW-CV according to the type of chemotherapy.

\begin{tabular}{lccccc}
\hline Cycle number & 1 & 2 & 3 & 4 & $\Delta \mathrm{C} 4 \mathrm{vs.C} \%$ \\
\hline 5-fluorouracil & 12 & 10.9 & 11.3 & 11.7 & -3 \\
Cisplatin & 11.8 & 10.9 & 10.7 & 10.4 & -12 \\
\hline
\end{tabular}

clearance is associated with increased odds of chemotherapyrelated toxicity (21).

Regarding the evolution of the MCV index, the tendency is upward, but with a period of latency or slight transient decrease. The latency period before MCV growth (or even a transient decrease) may extend over one or two treatment cycles (up to 6 weeks). Surprisingly, the decrease in Hb has no latency, and it is also maximal immediately after the first cycle. Furthermore, the cases with the highest increases of MCV in cycles 4 do not show latency in cycles 2 or 3 .

The evolution of the erythrocyte volume dispersion index (RDW-CV) is somewhat surprising regarding its reduction under chemotherapy, especially under cisplatin (correlated with the highest average MCV increase).

The average relative hemoglobin losses are between -2 and $-14 \%$ for the first 3 treatment cycles $(-1.39 \mathrm{~g} / \mathrm{dl}$ or a relative decrease of $-11 \%$ for all patient groups, statistically significant), with statistically significant differences also in hemoglobin levels between the sexes.

Risk factors associated with higher average losses are age $<50$ years or $>65$ years (statistically proven), BMI $>25$, cisplatin treatment (insufficient number of cases to reach statistical significance). Protective factors associated with lower losses are: Age between 50 and 65 years, BMI $<25 \mathrm{~kg} / \mathrm{m}^{2}$, limit values of creatinine $(<0.6 \mathrm{mg} / \mathrm{dl})$, chemotherapy treatments containing fluoropyrimidines (insufficient number of cases to reach statistical significance). The most substantial increases in MCV occur in persons $<50$ years of age, cisplatin treatment and in obese persons (insufficient cases for statistical significance).

\section{Acknowledgements}

Professional editing, linguistic and technical assistance was performed by Irina Radu, Individual Service Provider, certified translator in Medicine and Pharmacy (certificate credentials: Series E no. 0048).

\section{Funding}

No funding was received.

\section{Availability of data and materials}

All data and materials supporting the results of the present study are available in the published article. 


\section{Authors' contributions}

SS, DP, CO, AC, S,N conceived and designed the study. AN, MZ, IY, MP and S,N contributed to the interpretation of the results. SS was mainly responsible for the writing of the manuscript. All authors provided critical feedback and assisted with the design of the research, analysis and preparation of the manuscript. SS, DP, CO and SN designed the model and the computational framework, and analyzed the data. SS, CO and AC carried out the implementation. AN performed the calculations. SS and DP wrote the manuscript with input from all authors. AC and S,N conceived the study and were in charge of overall direction and planning. All authors read and approved the final manuscript.

\section{Ethics approval and consent to participate}

Not applicable.

\section{Patient consent for publication}

Not applicable.

\section{Competing interests}

The authors declare that they have no competing interests.

\section{References}

1. Eaves CJ and Eaves AC: Anatomy and physiology of hematopoiesis. In: Childhood Leukemias. 2nd edition. Pui CH (ed) Cambridge University Press, Cambridge, pp69-105, 2006.

2. Goodman SR, Kurdia A, Ammann L, Kakhniashvili D and Daescu O: The human red blood cell proteome and interactome. Exp Biol Med (Maywood) 232: 1391-1408, 2007.

3. Koury MJ: Erythroferrone: A missing link in iron regulation. The Hematologist. American Society of Hematology 12, 2015.

4. Kautz L, Jung G, Valore EV, Rivella S, Nemeth E and Ganz T: Identification of erythroferrone as an erythroid regulator of iron metabolism. Nat Genet 46: 678-684, 2014.

5. Wan J, Ristenpart WD and Stone HA: Dynamics of shear-induced ATP release from red blood cells. Proc Natl Acad Sci USA 105 16432-16437, 2008.

6. Diesen DL, Hess DT and Stamler JS: Hypoxic vasodilation by red blood cells: Evidence for an s-nitrosothiol-based signal. Circ Res 103: 545-553, 2008.

7. Kleinbongard P, Schutz R, Rassaf T, Lauer T, Dejam A, Jax T, Kumara I, Gharini P, Kabanova S, Ozüyaman B, et al: Red blood cells express a functional endothelial nitric oxide synthase. Blood 107: 2943-2951, 2006.
8. Bunn HF: Approach to the anaemias. In: Goldman-Cecil Medicine. Goldman L, Schafer AI (eds.) 25th edition. Philadelphia, PA: Elsevier Saunders, Chapter 158, 2016.

9. Chernecky CC and Berger BJ: Blood indices-blood. In: Laboratory Tests and Diagnostic Procedures. Chernecky CC and Berger BJ (eds). 6th edition. Elsevier, Philadelphia, PA, pp217-219, 2013.

10. Walker HK, Hall WD and Hurst JW (eds.): Clinical Methods: The History, Physical, and Laboratory Examinations. 3rd edition. Butterworths, Boston, 1990.

11. Evans TC and Jehle D: The red blood cell distribution width. J Emerg Med 9 (Suppl 1): S71-S74, 1991.

12. Rambach L, Bertaut A, Vincent J, Lorgis V, Ladoire S and Ghiringhelli F: Prognostic value of chemotherapy-induced haematological toxicity in metastatic colorectal cancer patients. World J Gastroenterol 20: 1565-1573, 2014.

13. Jung HA, Kim HJ, Maeng CH, Park SH, Lee J, Park JO, Park YS, Lim HY and Kang WK: Changes in the mean corpuscular volume after capecitabine treatment are associated with clinical response and survival in patients with advanced gastric cancer. Cancer Res Treat 47: 72-77, 2015.

14. Papaldo P, Ferretti G, Di Cosimo S, Giannarelli D, Marolla P, Lopez M, Cortesi E, Antimi M, Terzoli E, Carlini P, et al: Does granulocyte colony-stimulating factor worsen anaemia in early breast cancer patients treated with epirubicin and cyclophosphamide? J Clin Oncol 24: 3048-3055, 2006.

15. Groopman JE and Itri LM: Chemotherapy-induced anaemia in adults: Incidence and treatment. J Natl Cancer Inst 91: 1616-1634, 1999.

16. Ludwig $\mathrm{H}$ and Fritz E: Anaemia in cancer patients. Semin Oncol 25 (3 Suppl 7): S2-S6, 1998.

17. Stanca HT, Suvac E, Munteanu M, Jianu DC, Motoc AGM, Roşca GC and Boruga O: Giant cell arteritis with arteritic anterior ischemic optic neuropathy. Rom J Morphol Embryol 58: 281-285, 2017.

18. Balica NC, Poenaru M, Doros CI, Baderca F, Preda MA, Iovan VC, Stanca HT, Busuioc CJ, Oprişcan IC and Boruga O: The management of the oropharyngeal anterior wall cancer. Rom J Morphol Embryol 59: 113-119, 2018.

19. Pirker R, Pirolli M, Quigley J, Hulnick S, Legg J, Collins H and Vansteenkiste J: Hemoglobin decline in cancer patients receiving chemotherapy without an erythropoiesis-stimulating agent. Support Care Cancer 21: 987-992, 2013.

20. Mancuso A, Migliorino M, De Santis S, Saponiero A and De Marinis F: Correlation between anemia and functional/cognitive capacity in elderly lung cancer patients treated with chemotherapy. Ann Oncol 17: 146-150, 2006.

21. Peterson LL, Hurria A, Feng T, Mohile SG, Owusu C, Klepin HD, Gross CP, Lichtman SM, Gajra A, Glezerman I, et al: Association between renal function and chemotherapy-related toxicity in older adults with cancer. J Geriatr Oncol 8: 96-101, 2017.

This work is licensed under a Creative Commons Attribution-NonCommercial-NoDerivatives 4.0 International (CC BY-NC-ND 4.0) License. 